\section{Clinical genetics and pathology}

\section{G112 PATERNAL UPD14 ASSOCIATED WITH CONGENITAL CUTIS LAXA}

L.M. Cornette, A.B. Gill, D. Martinez, C. Hall, C.P. Bennett, C.J. Cullinane, S.M. Clark, D.W. Beverley, J.F. Harvey, D.T. Bonthron. Leeds General Infirmary, Department of Paediatrics, Leeds

We present the case of a male infant, born at 30 weeks GA, with facial dysmorphism (short palpebral fissures, protruding philtrum), bell-shaped thorax, extremely loose skin, large abdominal and bilateral inguinal hernias. Karyotype was normal. Defective elastin fibre formation observed on skin biopsy confirmed the clinical diagnosis of Cutis Laxa. A skeletal survey also revealed a narrow thorax with coat-hanger appearance of the ribs, short femurs and generalised osteopenia. So far, such skeletal dysplasia has not been reported in Cutis Laxa. Very recently, however, a new distinctive malformation complex due to paternal uniparental disomy of chromosome 14 (patUPD14) has been recognized in three patients, involving a strikingly similar constellation of thoracic hypoplasia, abdominal muscular defects and dysmorphic facies (Kurosawa et al. Am J Med Gen $2002 ; 110: 268)$. DNA collected from both parents and child using polymorphic markers confirmed patUPD1 4 in this case. Mechanical support was withdrawn at the age of six weeks due to severe cardiorespiratory failure.

Cutis Laxa can be caused by elastin mutations (chr 7) or can be X-linked (ATP7A mutation). No link with chr 14 has been identified so far. This is the first patient in which the phenotype of Cutis Laxa is directly linked with patUPD 14. Given the absence of Cutis Laxa in previously reported patUPD 14 patients, it seems unlikely that the presence of imprinted gene(s) on chr 14 would constitute the genetic mechanism of lethal Cutis Laxa. We therefore believe homozygosity for a recessive gene on chr 14 can account for this type of Cutis Laxa. Further work is planned on increasing the number of chr 14 markers that have been analysed, so as to define clearly the extent of heteroand iso-disomic regions. Also, this patient underlines the importance of DNA-analysis for patUPD 14 in any newborn with thoracic hypoplasia and loose skin.

\section{G113 10 YEAR FOLLOW UP OF A LARGE COHORT OF PATIENTS WITH NOONAN SYNDROME}

A.C. Shaw', E. Prappa', K. Kalidas', A.H. Crosby', S. Jeffery', W. McKenna', M.A. Patton'. 'Department of Medical Genetics and 'Department of Cardiological Sciences, St George's Hospital Medical School, London, UK

Clinical data on 151 individuals with Noonan syndrome was presented to this conference and published in the Archives of Disease in Childhood in 1992. This cohort has now been followed for over 10 years and up to date natural history data is presented.

Methods: Patients either attended St George's Hospital or were visited at home. Assessment included structured history taking, physical examination, with echocardiography and 3-dimensional facial photography where possible.

Results: The following results are given for the $50 \%$ of the cohort assessed so far. The proportion seen is expected to be $80 \%$ by the time of presentation. In those patients that have achieved their final height, average height was $171 \mathrm{~cm}$ in males $\left(9^{\text {th }}\right.$ to $25^{\text {th }}$ centile) and $153 \mathrm{~cm}$ in females $\left(2^{\text {nd }}\right.$ to $\left.9^{\text {th }}\right)$. A sub-cohort who received growth hormone showed increased growth velocity in the first year, but have yet to reach final height. Of those seen so far $68 \%$ have had no cardiac intervention at all, with $31 \%$ having had valvotomy or open heart surgery, $5 \%$ on more than one occasion and $5 \%$ have undergone heart transplantation. Severe feeding difficulties in infancy (requiring NG tube) are associated with special educational need in childhood $(p=0.01)$. Age at which children spoke with 2 word phrases was used as a marker for speech development and was on average delayed with a mean of 34 months. Of those who have left school $43 \%$ gained no qualifications although half of this group went on to life skills or vocational courses; $57 \%$ attained GCSE or equivalent qualifications; $19 \%$ went on to A-level and $17 \%$ to higher education. The majority of cases were either in full time employment or education with only $11 \%$ unable to work.

Conclusions: Noonan syndrome is associated with significant morbidity in childhood. In the long term however, the majority appear to have fewer problems and lead relatively normal adult lives.
G1 14 LETHAL MYELOPROLIFERATIVE DISORDER (CONGENITAL LEUKAEMIA) IN A NEONATE WITH THE KARYOTYPE OF BOTH DOWNS AND KLINEFELTERS SYNDROME

M. Sharma, E.S. Gray, D.J. Lloyd, D.J. King. Grampian University Hospitals NHS Trust, Aberdeen, UK

Myeloproliferative disorder of the newborn is a rare and usually transitory condtition, mostly associated with Downs syndrome (DS). Occasionally it is rapidly progressive and lethal (congenital leukaemia).

Born at 32 weeks gestation this infant presented with the DS phenotype, hepatosplenomegaly, bleeding problems, an omphalocoele, and hypoglycaemia. Peripheral blood showed anaemia and a very high white count $\left(211 \times 10^{\circ} / \mathrm{l}\right)$ of which $95 \%$ were blast forms. The bone marrow contained large numbers of blast cells derived from the erythoid, myeloid and megakaryocytic cell lines. Flow cytometry showed a profile of primitive myeloblasts with a strong megakaryoblastic component.

This is typical of the congenital myeloproliferative disorder associated with DS. Chromosomal analysis of blood and marrow confirmed the DS, but revealed an additional abnormality, 47XXY. The placenta $(1000 \mathrm{~g})$ was hydropic and the vessels contained blasts and erythoid cells with a nuclear change similar to that found in B19 infection. Titres for B19 and other viruses were negative.

Rapid deterioration in liver function, a rising white count and clotting problems resulted in chemotherapy, which provided transitory improvement. The development of a pericardial effusion suggested direct cardiac involvement and the infant died aged 6 weeks.

At autopsy the infant was hydropic, had a tense turbid pericardial effusion, patchy hepatic fibrosis and a very large spleen $(60 \mathrm{~g})$. The myocardium, liver, spleen and lymph nodes were infiltrated by primitive blast cells with megakaryoblasts being prominent. There was fibrosis of liver and pancreas. Skin fibroblasts confirmed the trisomy 21 (translocation 14:21) and 47, XXY as being constitutional. Klinefelter's has not been reported in association with neonatal myeloproliferative disorder.

\section{G115 HOW CAN WE IMPROVE THE ASCERTAINMENT OF CAUSE OF DEATH IN SUDDEN UNEXPECTED DEATH IN INFANCY?}

C. Okoro', N. Crowcroft ${ }^{2}$, on behalf of the Public Health Laboratory Service. 'Specialist Registrar in Public Health Medicine, Portsmouth City PCT; ${ }^{2}$ Consultant Epidemiologist, Immunisation division, CDSC, Colindale

The Public Health Laboratory Service (PHLS) has an interest in improving diagnosis of infection in sudden unexpected deaths in infancy (SUDI). This would extend use of advanced diagnostic methods to pathologists, bring standards of investigation of SUDI to that currently provided for infants in intensive care units, improve the estimation of the burden of vaccine preventable infections such as pertussis, pneumococcal and menigococcal disease, and improve the information on cause of death in mortality statistics and for parents. Mortality is a critical factor in evaluating vaccination programmes. For such rare diseases, surveillance of deaths through the medical certificate of cause of death has to be highly specific and sensitive for mortality statistics to be an accurate reflection of the true burden of disease.

SUDI still accounts for $27 \%$ of all infant deaths and it is unfortunate that a cause is found in only $20 \%$ of cases. The fall in Sudden Infant Death Syndrome (SIDS) incidence has been faster than the fall in infant mortality, and is not associated with any change in the characteristics of babies dying of SIDS. Indeed the characteristics of infants who die of SIDS are similar to those who die of explained causes in the SUDI population. These findings suggest that whatever factors were responsible for the fall in SIDS incidence did not change the underlying aetiology, and that the underlying cause of death in these infants may be among those already known. These observations together with the epidemiological, clinical and pathological features of SIDS are consistent with an infectious aetiology for some cases of SIDS and raise the possibility that precise diagnoses may be missed. It may also reflect a diagnostic shift or improvements in the specificity of death certification as a result of better diagnosis of infectious diseases.

Recent developments in highly sensitive molecular methods of diagnosing infection provide us with the opportunity to improve the investigation of SUDI. The implementation of such methods may be impeded by the lack of paediatric pathologists, issues related to the role of coroners, variation in regional arrangements, the implementation of the Health Protection Agency to replace the PHLS, and changes ensuing from various and numerous recent reviews and enquiries that 
will impact on the investigation and certification of cause of death. We will discuss the diagnostic methods that the PHLS (future HPA) provides, how these might be implemented into local protocols, and the organisational and professional changes that may help or hinder the process.

\section{G116 VALUE OF the NeONATAL Autopsy}

C. Wright, A.C. Fenton. Department of Pathology and Neonatal Unit, Royal Victoria Infirmary, Newcastle upon Tyne, NE 1 4LP, UK

Aim: Neonatal autopsy rates remain relatively high but are declining for many reasons including perhaps an increasing reliance on the validity of antemortem diagnosis and changing societal perceptions of the autopsy. The recent controversy surrounding organ retention has provide a further disincentive. The aim of this study was to assess the continuing clinical value of the neonatal autopsy.

Method: A retrospective view of all infants who underwent a specialist perinatal autopsy at a single centre in the Northern Region of England during 1999-2001, after receiving neonatal intensive care.
Autopsy findings were reviewed in the context of information provided in the discharge summary and postmortem request form, and cases allocated to one of three groups: (I) Confirmation of clinically suspected pathology; (II) Assessment of timing and severity of clinically suspected pathology; OR unexpected findings of clinical importance but not the major cause of death; (III) Major pathology causing/ contributing to death, only established at autopsy; OR resolution of other major clinical issues relating to death.

Results: 124 infant autopsies (89 neonatal deaths; 24 late neonatal; 11 post-neonatal) were reviewed. 61 cases (49\%) were allocated to group III, including: 11 infants (8.9\%) with other major malformations; 11 (8.9\%) dying from indiagnosed infection; and six $(4.8 \%)$ deaths due to iatrogenic disease. 42 autopsies $(34 \%)$ were allocated to group II, including $18(14.5 \%)$ providing information about the severity and timing of acquired CNS disease and three cases of indiagnosed kernicterus.

Conclusion: Neonatal autopsy continues to demonstrate clinically undiagnosed disease of importance both to families and clinicians. Neuropathology is very informative in this age group. 